\title{
Traffic Light Control System for Emergency Vehicles Using Radio Frequency
}

\author{
N. M. Z. Hashim 1 , A. S. Jaafar ${ }^{2}$, N. A. Ali ${ }^{3}$, L. Salahuddin ${ }^{4}$, N. R. Mohamad ${ }^{5}$, \\ M. A. Ibrahim 6 \\ 1,2,3,5,6 (Faculty of Electronics \& Computer Engineering, Universiti Teknikal Malaysia Melaka, Malaysia) \\ ${ }^{4}$ (Faculty of Information \& Communication Technology, Universiti Teknikal Malaysia Melaka, Malaysia)
}

\begin{abstract}
Traffic congestion problem is a phenomena which contributed huge impact to the transportation system in country. This causes many problems especially when there are emergency cases at traffic light intersections which are always busy with many vehicles. A traffic light controller system is designed in order to solve these problems. This system was designed to be operated when it received signal from emergency vehicles based on radio frequency (RF) transmission and used the Programmable Integrated Circuit (PIC) 16F877A microcontroller to change the sequence back to the normal sequence before the emergency mode was triggered. This system will reduce accidents which often happen at the traffic light intersections because of other vehicle had to huddle for given a special route to emergency vehicle. As the result, this project successful analyzing and implementing the wireless communication; the radio frequency (RF) transmission in the traffic light control system for emergency vehicles. The prototype of this project is using the frequency of $434 \mathrm{MHz}$ and function with the sequence mode of traffic light when emergency vehicles passing by an intersection and changing the sequence back to the normal sequence before the emergency mode was triggered. In future, this prototype system can be improved by controlling the real traffic situation, in fact improving present traffic light system technology.
\end{abstract}

Keywords: - PIC 16F877A, Radio Frequency (RF), Sequence Control, Traffic Light

\section{INTRODUCTION}

Many countries in the world are facing the problem at traffic light intersection that causes accident between emergency vehicle and other public vehicle. The traffic control system in Malaysia specifically has not been equipped with appropriate method when emergency case occurs. This will cause the emergency vehicles such as ambulances difficult to reach the destination on time because of the traffic congestion. Moreover, the situation is getting worse when emergency vehicles have to wait for other vehicles to give way at intersections with traffic lights. This causes a delay of time and may affect the emergency case. Besides, the collisions with other vehicles from other direction might occur at intersections when emergency vehicles had to override the red traffic lights. All these difficulties faced by emergency vehicles can be avoided using this traffic light control system based on radio frequency. Due to the problem, literature review for related issue prior to undertaking research project is decisive. The literature review will provide information on the technology available and methodologies used by other research counterparts around the world on this topic.

The traffic light system designed by Levi L. Rose [1] used only for emergency vehicle. Sensor is used to transmit signal that has been installed in every emergency vehicle to the receiver which has been placed at every traffic light intersection. When emergency vehicle reach at the traffic light intersection, the signal code will be sent information of frequency modulation to the receiver. The receiver demodulates the received code and the red traffic light will trigger at all the junctions. Thus, emergency vehicle will have special route from other vehicle to reach the destination.

The traffic light system designed by M. R. Smith et al [2] provided early warning of the approaching an emergency vehicle to find a way out from traffic congestion and lead the emergency vehicle to the destination. The emergency vehicle also may take control of traffic light at an intersection. A transmitter placed on an emergency vehicle transmits a signal to the receivers positioned at the traffic lights whenever it is on emergency mode. The received signal is then processed by a master controller which in turn pre-empts the sequence of the traffic light to control the traffic flow at the intersection which taken by the emergency vehicle. The master controller also provides an output which display signs to indicate that there is an emergency vehicle to the other road users from other direction at the traffic light intersection. Additionally, the display system indicates whether the emergency vehicle has passed through the intersection or not. 


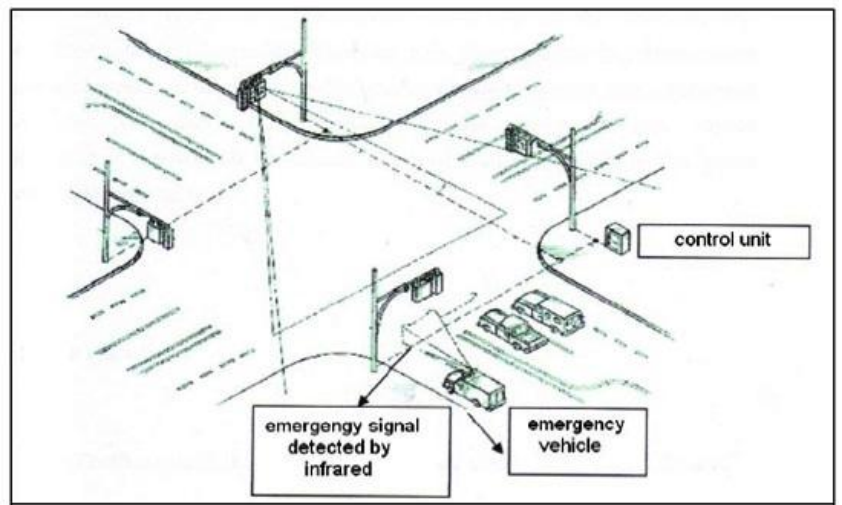

Fig.1 Four-traffic light intersection traffic light [2]

W. L. Mitchell has designed a traffic light control system [3] which had overcame the traffic congestion problem and provided an emergency path for the emergency vehicle where the radio transmitter and antenna placed on the emergency vehicle. The radio will transmit the signal to the other vehicle that nearby. The radio receiver had been placed at four junction traffic light will receive the emergency signal from emergency vehicle that passed by the junction. The first signal code contains a frequency for emergency vehicle while the second signal code contains a frequency for other vehicle. The transmitted signals provide miscellaneous traffic light pole in normal condition or emergency. When the receiver received the signal from emergency vehicle transmitter, traffic light system for emergency vehicle will be activated.

W. E. Brill introduced an emergency vehicle detection system [4] for alerting a driver of an approaching emergency vehicle includes a sound signal-producing unit mounted on an emergency vehicle, a sound signal detection unit mounted on a non-emergency vehicle, and a display unit remotely located on the non-emergency vehicle. The sound signal-producing unit has a sound generator for producing and transmitting a sound signal. A switch is used for controlling the operation of the sound generator in combination with a siren.

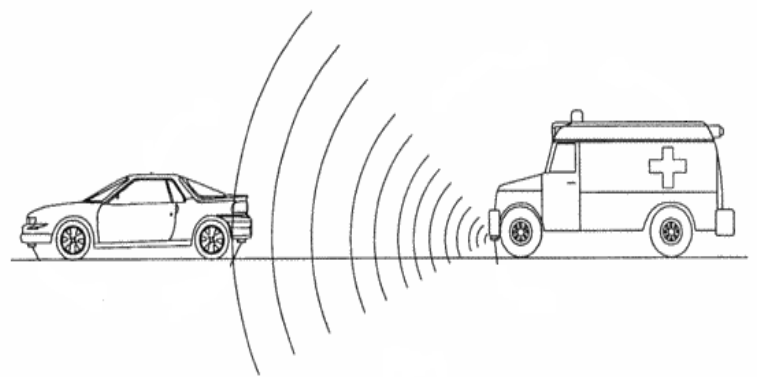

Fig. 2: Sound signal being transmitted [4]

The sound signal detection unit has at least one sound transducer for detecting sound signals and producing an electric current upon detection of a signal. A signal comparator is connected to the sound transducers for comparing the currents from the transducers to pre-programmed patterns. If there is matching pattern, a signal output encoder connected to the signal comparator constructs an encoded signal and transmits the encoded signal to a remotely located display unit through a transmitter. The display unit has a receiver for receiving the encoded signal and passing it to a signal comparator to compare the encoded signal to known patterns and activate at least one illumination device upon detection of a matched pattern.

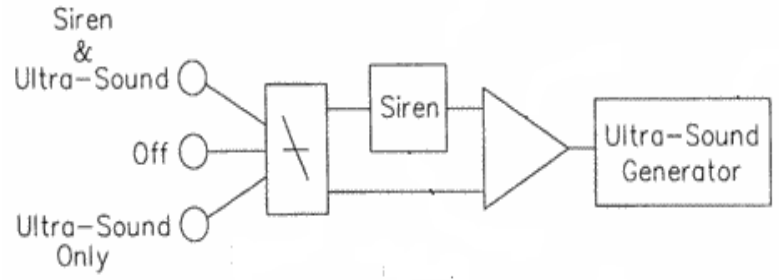

Fig. 3 The block diagram [5] 
A traffic light control system invented by Carl J. Obeck [5] consists of two-way communication between emergency vehicles approaching a busy intersection with one or more traffic lights. The system temporarily pre-empt the sequence of the traffic light and provides the most effective method of routing the vehicle through the intersection while redirecting general traffic. As part of the invention, the traffic light control system will inform the emergency vehicle which it has received the transmitted signal. The stored preset traffic patterns may in one representation is responsive to manual intervention from a dispatching centre or to time-of-day conditions. The traffic light control apparatus may be operated under control of data or voice transmitted from the emergency vehicle's regular two-way voice communications system to a central control station.

According to all these papers, a convenient wireless communication between emergency vehicles and the traffic light is by using RF. The prototype of this project is using the radio frequency of $434 \mathrm{MHz}$ compared to the range of about $3 \mathrm{kHz}$ to $300 \mathrm{GHz}$ of frequency which have been reserved for the RF theoretically. There are three objectives to be achieved in this project. First is to analyze and implement wireless communication; Radio Frequency (RF) transmission system in traffic light control system for emergency vehicles. Second is to design a traffic light sequence for emergency mode when receive signal from emergency vehicles. Last objective is to change the sequence back to the normal sequence before the emergency mode was triggered. This project has contributed in implementing the wireless communication by using the radio frequency (RF) transmission of $434 \mathrm{MHZ}$ in the traffic light control system for emergency vehicles.

\section{MATERIALS AND METHODS}

\subsection{Flow chart}

\subsection{Component Consideration}

The information and data for component consideration are based on the reviewed journal and patents. All type of components must be consider first in order to ensure that it is suitable for this project.

\subsection{Choosing Component}

After consideration is done, there is a section where the component should be look and fully understood about their advantages and disadvantages. The component must be chosen properly before it will be proceed to design process. If the component is not compatible due to disadvantages, then it will go back to the consideration component process again.

\subsection{Configuration Design}

The circuit of this project is designed and constructed roughly using the entire chosen component during this phase. The components are assembled on a breadboard to ensure that the circuit work properly.

\subsubsection{Radio Frequency Transmission}

A radio frequency $(\mathrm{RF})$ signal begins as an electrical alternating current (AC) signal that is originally generated by a transmitter [6]. This AC signal is sent via a copper conductor which usually a coaxial cable and radiated out of an antenna element in the form of an electromagnetic wave. Changes of current flow in the antenna produce changes in the electromagnetic fields around the antenna.

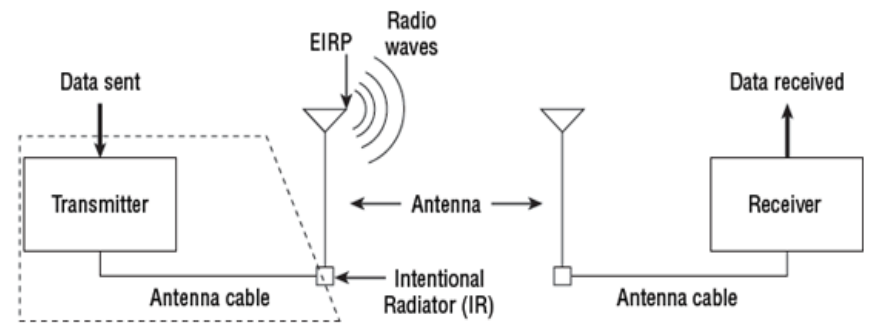

Fig. 4 The RF components [6]

\subsubsection{RF Based Wireless Remote using TX-RX Modules}

This circuit utilizes the RF module, transmitter and receiver (TX-RX) for making a wireless remote, which could be used to drive an output from a distant place [7]. RF module, as the name suggests, uses radio frequency to send signals. These signals are transmitted at a particular frequency and a baud rate. A receiver can receive these signals only if it is configured for that frequency. A four channel encoder/decoder pair has also been used in this system. The input signals, at the transmitter side, are taken through four switches while the outputs are monitored on a set of four light emitting diodes (LED) corresponding to each input switch. The 
circuit can be used for designing remote appliance control system. The outputs from the receiver can drive corresponding relays connected to any household appliance.

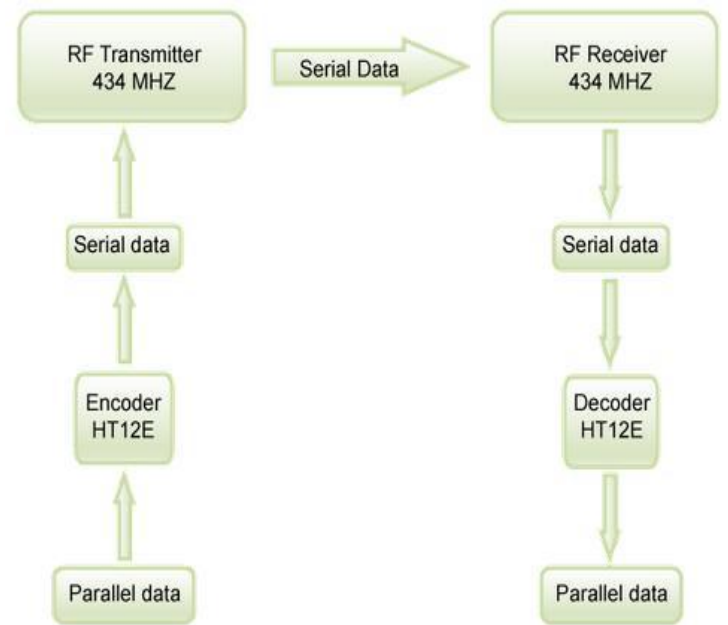

Fig. 5 Tx-Rx module block diagram [7]

This RF transmission system employs Amplitude Shift Keying (ASK) with transmitter/receiver $(\mathrm{Tx} / \mathrm{Rx})$ pair operating at $434 \mathrm{MHz}$. The transmitter module takes serial input and transmits these signals through RF. The transmitted signals are received by the receiver module placed away from the source of transmission. The system allows one way communication between two nodes, namely, transmission and reception.

The RF module has been used in conjunction with a set of four channel encoder/decoder ICs. The encoder converts the parallel inputs (from the remote switches) into serial set of signals. These signals are serially transferred through RF to the reception point. The decoder is used after the RF receiver to decode the serial format and retrieve the original signals as outputs. These outputs can be observed on corresponding LEDs.

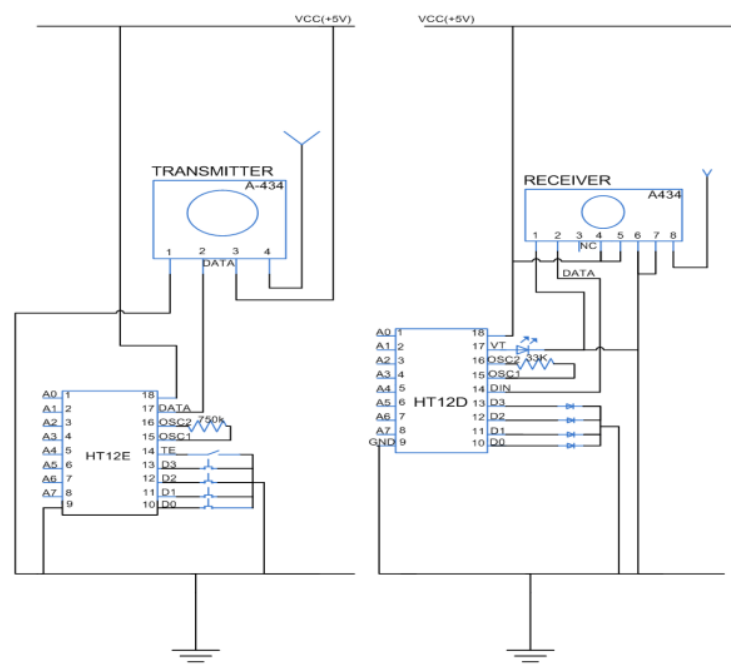

Fig. 6 RF (Tx-Rx) module [8]

Transmission through RF is better than infrared (IR) because of many reasons. Firstly, signals through $\mathrm{RF}$ can travel through larger distances making it suitable for long range applications. Besides, while IR mostly operates in line-of-sight mode, RF signals can travel even when there is an obstruction between transmitter \& receiver. Next, RF transmission is more strong and reliable than IR transmission. RF communication uses a specific frequency unlike IR signals which are affected by other IR emitting sources.

The RF module comprises of an RF Transmitter and an RF Receiver. The transmitter/receiver (Tx/Rx) pair operates at a frequency of $434 \mathrm{MHz}$. An RF transmitter receives serial data and transmits it wirelessly through RF through its antenna connected at pin 4 . The transmission occurs at the rate of $1 \mathrm{Kbps}-10 \mathrm{Kbps}$. The transmitted data is received by an RF receiver operating at the same frequency as that of the transmitter [7]. 


\subsection{Complete Design}

After all drawing, assemble and analysis process, the complete design can be viewed using a Proteus software. Complete design must be carefully checked in order to find the defect in the design. If there is any defect, it must be returned back to configuration design process.

\subsection{Finalize Design}

Complete design must be finalized before going to the end of all the processes in the project. The design system will be rechecked to ensure the design is valid for this project to be run successfully.

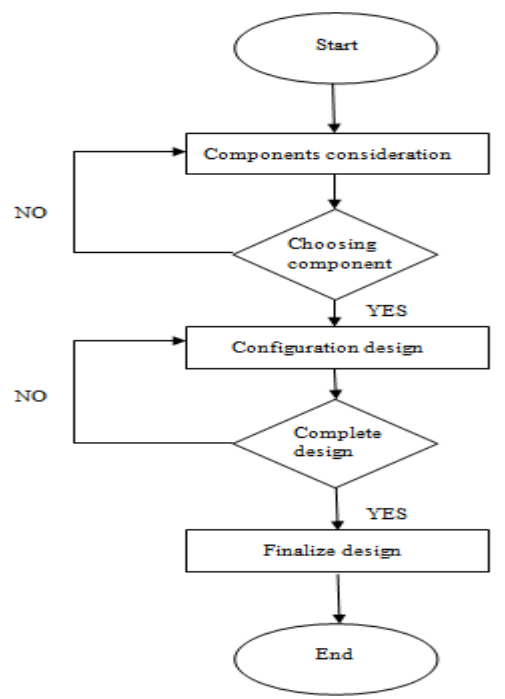

Fig.7 Project flowchart

\subsection{Project Block Diagram}

The block diagram of this project is shown in Fig. 8.The block diagram is divided into several parts such encoder, RF transmitter and receiver, decoder and embedded microcontroller.

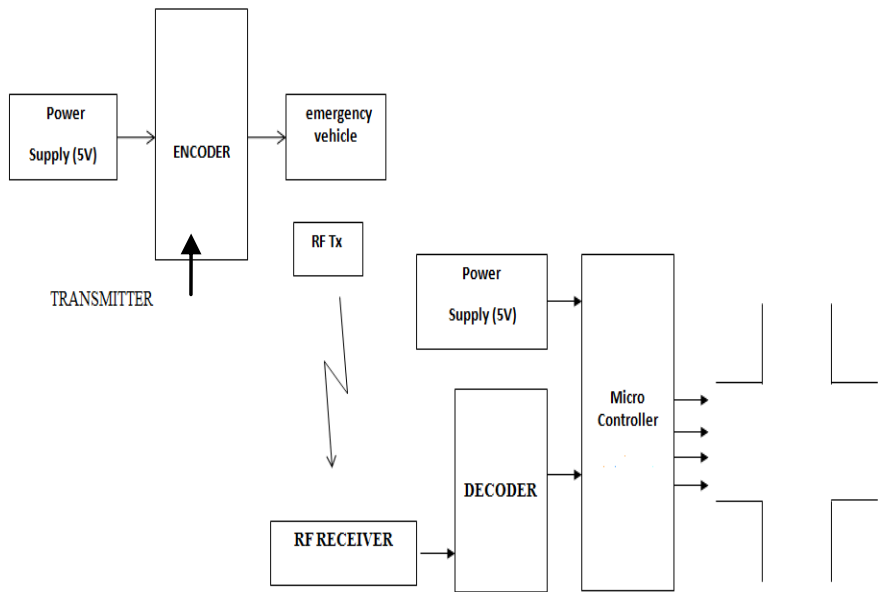

Fig. 8 Project block diagram Traffic Light Intersection

\subsection{Encoder}

Encoding is a popular way of securing data and information; changing the format so that it can only be read by someone with the appropriate decoding equipment or software. An encoder is a device or entity that will encode information in a particular way, compressing, converting or securing it into a different format. Since there are four traffic lights at the intersection, multiple channel encoders will be used for this project [9].

\subsection{Decoder}

The encoding process essentially scrambles all the pieces of the data up and they are then put back together at the other end by the decoder. A decoder is the device or entity that will remove the information from 
its previously encoded state and return it to its original format. The decoder must have the same number of channel with the encoder [9].

\subsection{RF Transmitter and Receiver Module}

A radio frequency transmitter and receiver module will be used in the circuit to implement wireless communication for this project.

\subsection{Embedded Microcontroller}

PIC 16F877A, a 40-pins PIC will be used to operate the end device system since it has many ports and most importantly is supports UART features.

\section{RESULTS / FINDING AND DISCUSSION}

This project is divided into two parts of electronic circuit. First part is the RF transmitter circuit which can override the sequence of the traffic light. Meanwhile, the second part is the RF receiver and the microcontroller circuit that generate the traffic light sequences for a four-traffic lights intersection.

\subsection{RF Transmitter Circuit}

The transmitter circuit consists of a RF transmitter module, an encoder, a set of four push-on switch and a power supply. The connection of the circuit is shown below.

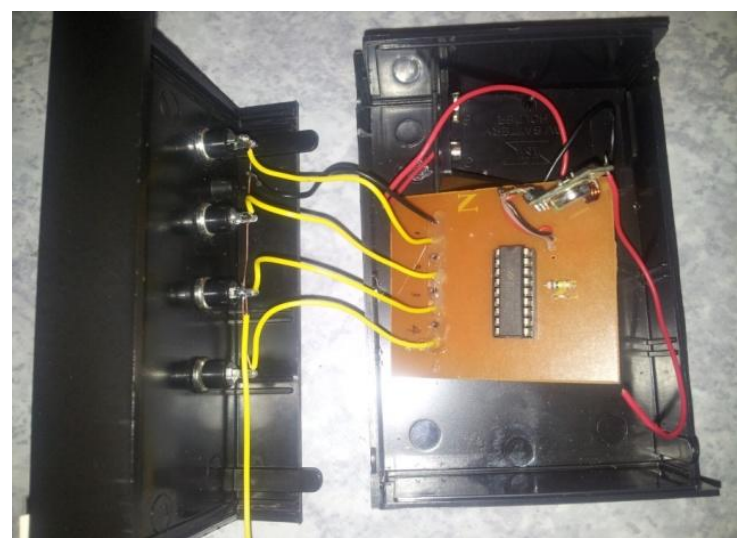

Fig. 9 RF transmitter circuit

\subsection{Push-ON Switches}

A set of four push-on switches is used in the RF transmitter circuit. Each switch labeled with number 1, 2,3 , and 4 to indicate which traffic light at the intersection. These switch need to be push (switch on) in order to trigger the emergency sequence mode of the traffic light intersection.

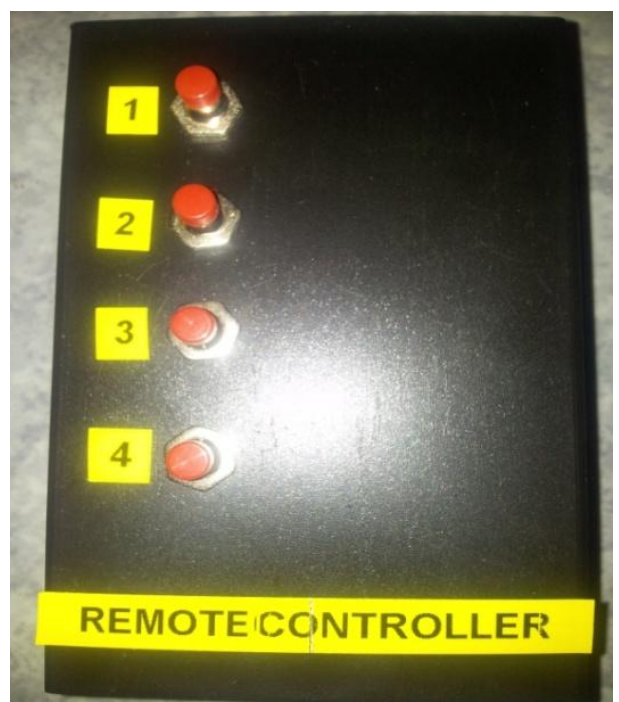

Fig. 10 Four push-on switch 
When any of these switch is being pushed-on, the RF transmitter send a signal that have been coded to the receiver which then will be used to override the traffic light sequence.

\subsection{RF Receiver Circuit}

The $\mathrm{RF}$ receiver circuit consists of a RF receiver module, a decoder, relays and LEDs. A LED is connected to pin 14 of the decoder to indicate that the RF transmitter is in the range with the RF receiver. A LED is connected to each relay to indicate which traffic light has been triggered by the signal transmitted from the RF transmitter.

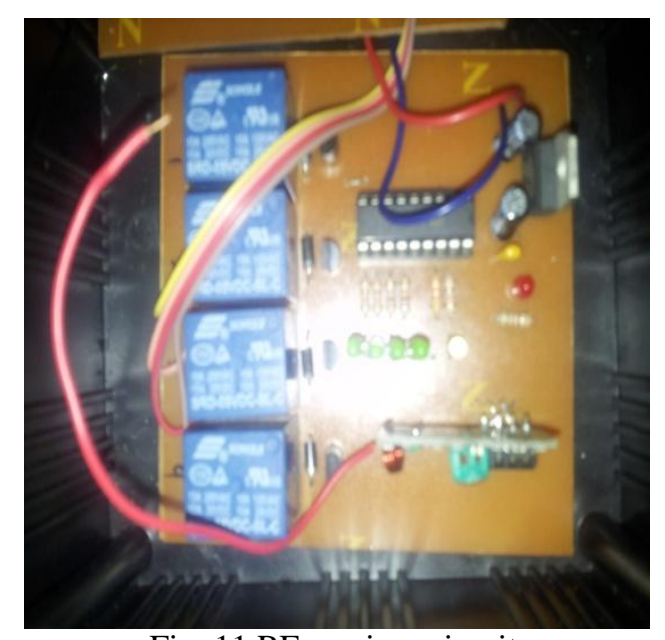

Fig .11 RF receiver circuit

\subsection{Traffic Light Circuit}

A circuit of four traffic light intersection has been designed for this project using a PIC 16F877A microcontroller [10]. The sequence of the traffic lights is generated by the PIC 16F877A. The input from the RF receiver circuit has been used to override the sequence.

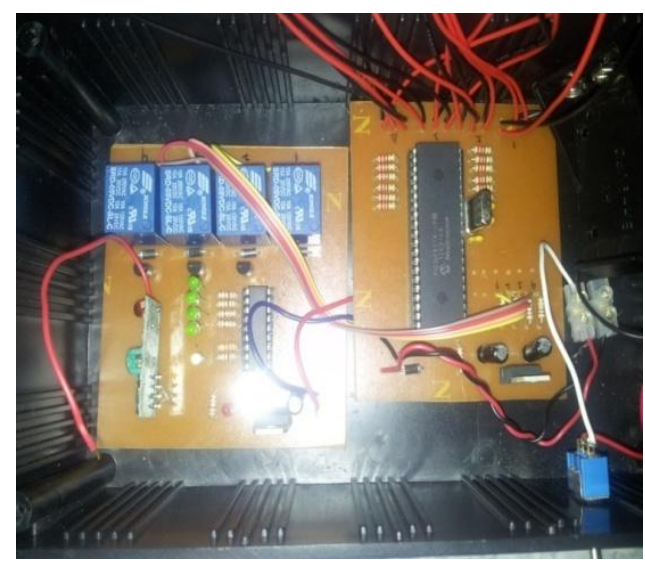

Fig. 12 Traffic light circuit

\subsection{The Traffic Light Sequences}

\subsection{The Normal Sequence}

The sequence of the traffic lights started as green light of traffic light 1 and red light of other traffic lights are on. The duration for this mode lasted for 30 seconds unless the RF receiver triggers any signal from the transmitter to override the sequence.

Table 1 Truth Table for First 30 Seconds of the Sequence

\begin{tabular}{|c|c|c|c|}
\hline Traffic Light & Red & Yellow & Green \\
\hline 1 & 0 & 0 & 1 \\
\hline 2 & 1 & 0 & 0 \\
\hline 3 & 1 & 0 & 0 \\
\hline 4 & 1 & 0 & 0 \\
\hline
\end{tabular}




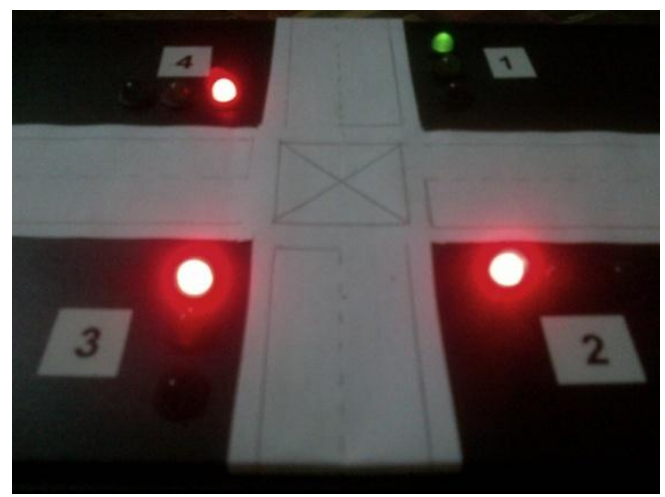

Fig. 13 The green light of traffic light 1 is on for 30 seconds

Then, the green light of traffic light 1 is off and the yellow light of the same traffic light is on for 2 seconds.

Table 2 Truth Table When the Yellow Light of Traffic Light 1 Is On

\begin{tabular}{|c|c|c|c|}
\hline Traffic Light & Red & Yellow & Green \\
\hline 1 & 0 & 1 & 0 \\
\hline 2 & 1 & 0 & 0 \\
\hline 3 & 1 & 0 & 0 \\
\hline 4 & 1 & 0 & 0 \\
\hline
\end{tabular}

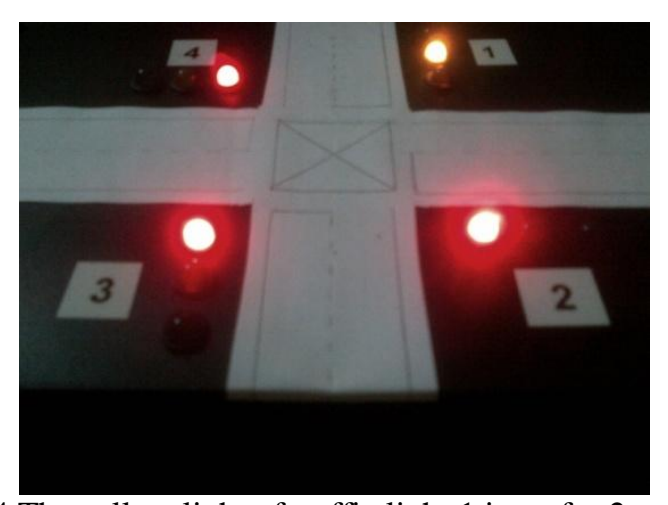

Fig.14 The yellow light of traffic light 1 is on for 2 seconds

Next, the sequence turned on the green light of traffic light 2 and the red light of other traffic light for duration of 30 seconds. The same thing happened to the traffic light 3 and traffic light 4 after an interval of the yellow light of each traffic light is on for 2 seconds. The microcontroller will keep repeating this sequence of the traffic light unless the emergency sequence is triggered.

\subsection{The Emergency Mode Sequence}

The emergency mode is triggered when the RF receiver received the transmitted signal from the RF transmitter to override the normal sequence of the traffic light. For example, an ambulance arrives at the traffic light 4 and the green light of the traffic light 1 is on.

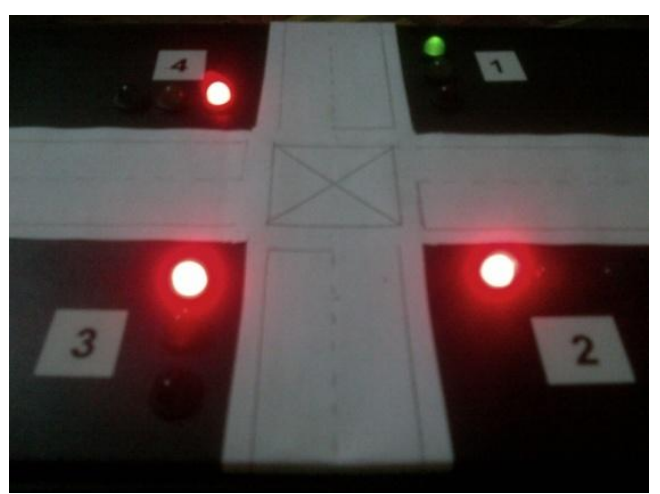

Fig. 15 The green light of traffic light 1 is on 
When the push-on button no.4 is turned on, the RF receiver received the transmitted signal and changed the sequence to the emergency sequence mode. The emergency sequence mode started when the yellow of traffic light 1 is on for 2 seconds. Then the green of traffic light 4 is on for 10 seconds and then the yellow light of the same traffic light is turned on for 2 seconds.

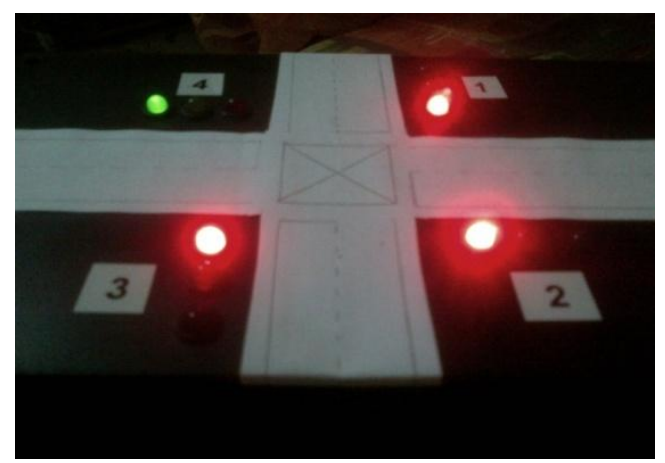

Fig. 16 The green light of traffic light 4 is on for 10 seconds

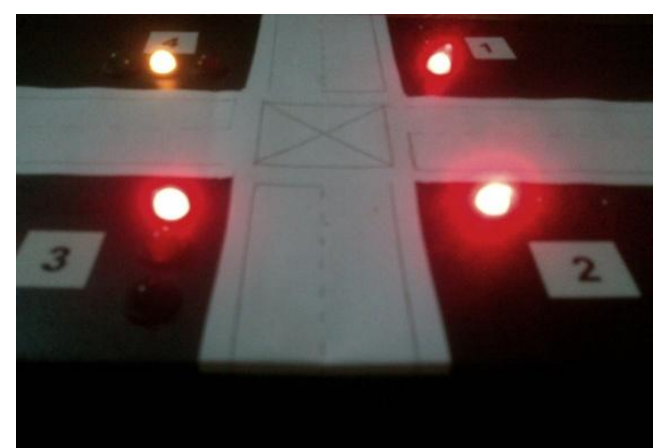

Fig. 17 The yellow light of traffic light 4 is on for 2 seconds

The emergency sequence mode is ended when the sequence of the traffic light is back to the normal sequence which the green light of traffic light 1 is turned back on for the remaining time before the emergency sequence mode is triggered.

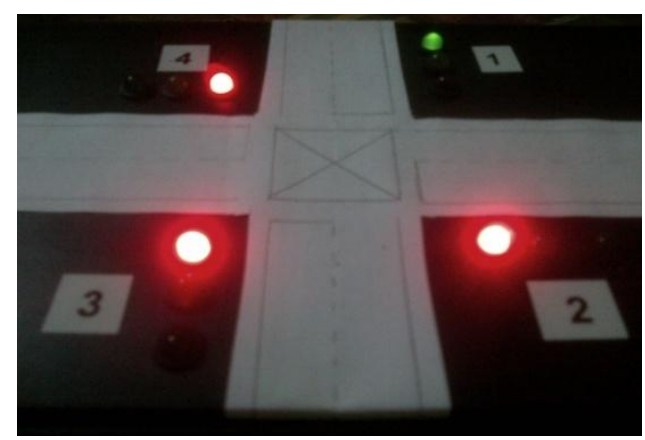

Fig. 18 The sequence of the traffic light is back to normal

\section{IV.}

\section{CONCLUSION}

As a conclusion, this project have achieved the main objective stated earlier which is analyzing and implementing the wireless communication; the radio frequency (RF) transmission in the traffic light control system for emergency vehicles. The prototype of this project is using the frequency of $434 \mathrm{MHz}$ compared to the range of about $3 \mathrm{kHz}$ to $300 \mathrm{GHz}$ of frequency which have been reserved for the RF theoretically. Besides, the functionality of this project proved that the other objectives have been successfully attained which are designing an emergency sequence mode of traffic light when emergency vehicles passing by an intersection and changing the sequence back to the normal sequence before the emergency mode was triggered. The sequences for this project have been developed using the programming in the microcontroller PIC 16F877A. In future, this prototype system can be improved by controlling the real traffic situation and the study can be done by investigating the length, reception and transmission issue for the system to be operated with this traffic light system. 


\section{ACKNOWLEDGEMENTS}

We are grateful to Universiti Teknikal Malaysia Melaka (UTeM) for their kind help for supplying the electronic components and giving their laboratory facility to complete this study.

\section{REFERENCES}

[1] Levi L. Rose, "Emergency Traffic Control System with Security Transmission Coding", United States Patent, April 5th, 1997.

[2] Michael R. Smith, Paul J. Davidson and Henry L. Pfister, "Emergency Vehicle Warning and Traffic Control System”, United States Patent, October 4th, 1998.

[3] Willbur L. Mitchell, "Traffic Light Control For Emergency Vehicles", United States Patent, April 17th, 1994.

[4] William E. Brill, "Emergency Vehicle Detection System”, United States Patent, March 26th, 2002.

[5] Carl J. Obeck, "Traffic Signal Control For Emergency Vehicles", United States Patent, May 7th, 1998.

[6] David D. Coleman, David A. Westcott, "CWNA: Certified Wireless Network Administrator Official Study Guide”, Sybex, April 06, 2009.

[7] Mir Roomi Rahil, Rajesh Mahind, Saurabh Chavan, Tanumay Dhar, "GLCD-Touchpad Based Restaurant Ordering \& Automatic Serving System", International Journal of Recent Technology and Engineering (IJRTE), 2013.

[8] "RF Based Wireless Remote using Tx-Rx Modules", Chawla Radios \& Electricals, August 11th, 2011.

[9] http://www.blurtit.com/q335716.html, What Is The Difference Between Decoder And Encoder? Lukas Hoffmann, "PIC 16F877A Tutorials for Pitt Robotics Club", 2010.

[10] http://www.microchip.com, PIC16F87XA Data Sheet, Microchip Technology Inc. 